\title{
Predictors of good response to conventional synthetic DMARDs in early seronegative rheumatoid arthritis: data from the ESPOIR cohort
}

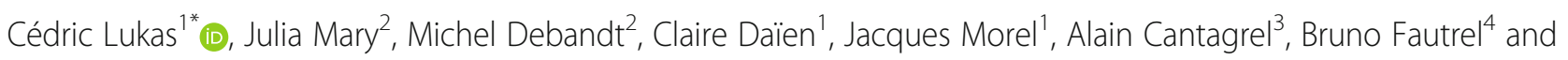
Bernard Combe ${ }^{1}$

\begin{abstract}
Background and objective: Early seronegative rheumatoid arthritis (RA) is considered a specific entity, especially regarding diagnostic issues and prognosis. Little is known about its potentially different initial clinical presentation and outcome. We aimed to determine predictors of good response to conventional synthetic disease-modifying anti-rheumatic drugs (csDMARDs) in seronegative RA patients with early inflammatory arthritis.

Patients and methods: Patients from the ESPOIR cohort with early inflammatory arthritis fulfilling the 2010 ACR/ EULAR classification criteria for RA despite negativity for both rheumatoid factor and anti-CCP antibodies. The primary endpoint was a good or moderate EULAR response assessed after 1 year of follow-up, given at least 3 months of treatment with a csDMARD. Secondary objectives were to compare the early therapeutic response to methotrexate (MTX) and leflunomide (LEF) versus other csDMARDs (hydroxychloroquine, sulfasalazine) and to identify factors associated with functional disability (Health Assessment Questionnaire-Disability Index [HAQ-DI] >0.5 at 1 year) and structural progression (van der Heijde-modified total Sharp score $>1$ and $>5$ points at 1 year). Logistic regression analysis was used to determine independent predictors of outcomes.
\end{abstract}

Results: One hundred seventy-two patients were analyzed. Overall, 98/172 (57\%) patients received MTX during the first year of follow-up. A good or moderate EULAR response at 1 year was associated with early use of cSDMARDs (i.e., within 3 months after the first joint swelling) on univariate and multivariable analysis (odds ratio $=2.41$ [95\% confidence interval 1.07-5.42], $p=0.03$ ). Response rates were not affected by other classical prognostic factors (i.e., baseline DAS28). Presence of erosions at baseline was associated with Sharp score progression $>1$ point and $>5$ points (both $p=0.03$ ) at 1 year. HAQ-DI $\geq 1$ at inclusion and active smoking were significantly associated with HAQ$\mathrm{DI}>0.5$ at 1 year.

Conclusion: Our results suggest that delay in initiation of CsDMARD more than baseline clinical, biological, or imaging features predominantly affects the outcome in early seronegative RA. These findings confirm that the usual therapeutic concepts in RA (early treatment, tight control, and treat-to-target) should be applied similarly to both seropositive and seronegative disease forms.

Trial registration: ClinicalTrials.gov: NCT03666091. Registered September 11, 2018.

Keywords: Rheumatoid arthritis, Seronegative, ESPOIR cohort, DMARD

\footnotetext{
*Correspondence: c-lukas@chu-montpellier.fr

${ }^{1}$ Rheumatology Department, CHU Montpellier, Montpellier University,

Montpellier, France

Full list of author information is available at the end of the article
}

(c) The Author(s). 2019 Open Access This article is distributed under the terms of the Creative Commons Attribution 4.0 International License (http://creativecommons.org/licenses/by/4.0/), which permits unrestricted use, distribution, and reproduction in any medium, provided you give appropriate credit to the original author(s) and the source, provide a link to the Creative Commons license, and indicate if changes were made. The Creative Commons Public Domain Dedication waiver (http://creativecommons.org/publicdomain/zero/1.0/) applies to the data made available in this article, unless otherwise stated. 


\section{Background}

Rheumatoid arthritis (RA) is a chronic autoimmune disease affecting about $0.4 \%$ of the general population [1]. Rheumatoid factor (RF) and anticitrullinated protein antibodies (ACPA) are the most relevant antibodies associated with RA, and their testing is valuable early in the disease course. However in early disease, antibody detection has been reported as low as 50\% [2].

RF and ACPA status are important for both the diagnosis and prognosis of RA [3, 4]. Seropositive RA, particularly ACPA-positive status, is associated with increased likelihood of the development of erosions and further radiographic progression [5-7]. Less is known about the clinical presentation and outcomes of seronegative RA, and studies are disparate given that seronegative RA is more challenging to classify and may indeed represent a heterogeneous population.

Early and intensive treatment of RA with diseasemodifying antirheumatic drugs (DMARDs) is clinically beneficial $[8,9]$. The difficulty in reliably diagnosing RA presumably explains the discrepancy between general guidelines and daily practice: although methotrexate (MTX) should be prescribed as soon as RA is diagnosed, it is less often prescribed to "seronegative" patients and mostly as a single treatment [10]. Furthermore, the association between the presence of autoantibodies and response to treatment is controversial [11-13]. Because seronegative RA is thought to represent a separate entity with presumably different pathogenesis and less severe disease, assessing the differences in response to treatment with seronegative RA would be useful for physicians.

To our knowledge, no study has specifically assessed seronegative patients in terms of response to treatment. Therefore, we aimed to describe characteristics of seronegative RA disease at presentation and assess factors associated with good response to specific treatment and whether the choice of the first prescribed DMARD might influence the short-term clinical evolution (at 1 year).

\section{Methods}

\section{Objectives}

For this study, we used data from a French longitudinal prospective cohort of adult patients with early arthritis, the Etude et Suivi des POlyarthrites Indifferenciées Récentes (ESPOIR) cohort [14], to determine predictors of good response to conventional DMARDs at 1 year in seronegative RA patients. Secondary objectives were to compare the early therapeutic response to MTX and leflunomide (LEF) versus other conventional synthetic DMARDs (csDMARDs; hydroxychloroquine, sulfasalazine) and to identify factors associated with functional disability (Health Assessment Questionnaire-Disability
Index [HAQ-DI] $>0.5$ at 1 year) and structural progression (van der Heijde-modified total Sharp score [mTSS] $>1$ point and $>5$ points at 1 year).

\section{Study population}

The ESPOIR cohort is a nationwide prospective cohort study of adults conducted under the umbrella of the French Society of Rheumatology. The cohort was constituted by asking general practitioners and rheumatologists to refer patients with early arthritis to one of the 14 university hospitals participating in the ESPOIR cohort project. The protocol has been described in detail elsewhere [14] (ClinicalTrials.gov NCT03666091). Briefly, patients were eligible if they had a definite or probable clinical diagnosis of RA or a diagnosis of undifferentiated arthritis with potential for progression to RA. Patients were included if they were 18 to 70 years old and had swelling of $\geq 2$ joints for 6 weeks, symptom duration $<6$ months, and no prior treatment with DMARDs or glucocorticoids. Patients with another definite diagnosis of an inflammatory rheumatic disease at the baseline visit were excluded. Included patients were evaluated every 6 months for 2 years, then once a year for at least 10 years. Each center acted as an observational center and did not interfere with patient treatment unless it was in charge of the patient. The patients were routinely monitored and followed by private rheumatologists in the geographic area. Between November 2002 and April 2005, 813 consecutive patients were included in the ESPOIR cohort. Patients were included in the current analysis if they fulfilled the 2010 American College of Rheumatology/European League Against Rheumatism (ACR/ EULAR) classification criteria for RA [15], were seronegative for both ACPA and RF, and had been prescribed at least 1 DMARD over the first year period of follow-up; all other individuals, defined as seropositive, were excluded.

\section{Baseline assessment}

We collected data on demographics (age, sex); socioeconomic status; education (primary, middle, or high school or university); tobacco exposure; duration of symptoms at first visit (defined by the first fixed swollen joint); clinical features [number of swollen joints (0-28); number of tender joints (0-28); visual analog scale (VAS; 0-100) overall assessment by the physician; Disease Activity Score in 28 joints (DAS28) [16]; functional disability by the HAQ-DI [17]]; therapeutic regimen; biological features [including erythrocyte sedimentation rate $(\mathrm{ESR} ; \mathrm{mm} / \mathrm{h})$; level of $\mathrm{C}$ reactive protein $(\mathrm{CRP} ; \mathrm{mg} / \mathrm{l})$ by standard laboratory methods]; radiographs of hands, wrists, and feet in the posteroanterior view; and therapeutic regimen. 
Radiographs of the hands, wrists, and feet were scored for the presence of erosions and joint space narrowing according to the mTSS [18] by an experienced rheumatologist who was blinded to the patient's other data.

\section{Follow-up assessment and outcomes}

The primary endpoint was a EULAR response classified as good or moderate versus none evaluated at 1 year, given at least 3-month csDMARD treatment over the first year of follow-up had been followed. Patients with at least 3 months of a first csDMARD but who switched to a second drug were considered non-responders to the first treatment. Because the reason for the switch was not specifically collected in ESPOIR, we considered that a very early withdrawal (within 1 month) of a csDMARD was presumably due to intolerance or side effects. In the latter situation, the second csDMARD used was considered the first-line therapy. We also considered patients with more than 1 month but no longer than 3-month treatment with a csDMARD as non-responders. All patients were followed for at least 1 year. Radiographs were obtained and scored at 12 months in a chronological order by using the same technique. Radiographic progression was defined as an increase of at least 1 point of the mTSS (the smallest detectable change derived from this scoring) or the erosion score assessed at baseline and after 12 months [19].

\section{Statistical analysis}

Baseline characteristics and disease evolution are described with mean \pm SD or frequency (\%) as appropriate. Baseline characteristics and disease course were compared between good or moderate EULAR responders and non-responders by the Mann-Whitney $U$ test (for numerical data) and Fisher exact test (for categorical data). Logistic regression analyses were used to determine relevant independent baseline variables, estimating odds ratios (ORs) and 95\% confidence intervals (CIs). The explanatory variables included in the logistic regression model were derived from results of univariate analyses. Significance was defined as $p<0.05$ for variables in the final multivariable model. A similar approach was used to analyze the association of baseline variables with the secondary outcomes (i.e., radiographic progression [change in mTSS at least 1 point and at least 5 points] and functional impairment at 1 year [HAQ-DI >0.5]). To compare the early therapeutic response to MTX and LEF versus other csDMARDs (hydroxychloroquine, sulfasalazine), a propensity score was used to reduce confounding by indication bias. Indeed, the likelihood of prescribing MTX or LEF rather than hydroxychloroquine or sulfasalazine is influenced by the appreciation by the rheumatologist of the disease severity and activity at baseline. The propensity score was computed by using a multivariable logistic regression model. All demographic and disease characteristics at baseline were used as covariates in the model: sex, age at inclusion, ESR, CRP level, tender and swollen joint count, disease activity, VAS score, HAQ-DI, erosive disease, erosion score, and joint narrowing score. As sensitivity analyses, we conducted the same analysis plan in the entire ESPOIR cohort, restricted to patients fulfilling ACR/EULAR 2010 criteria for RA and having been treated by at least 1 DMARD in their first year of follow-up. We also tested alternative outcome measures than EULAR response in seronegative patients, with DAS28 remission and DAS28 remission/low disease activity at 12 months being used. Statistical analysis involved using SPSS v15. $P<0.05$ was considered statistically significant.

\section{Results}

\section{Patient characteristics}

From the 813 patients included in the ESPOIR cohort, 24 had missing data regarding RF-and CCP-tests. Of the 789 with available data, 384 (48.7\%) were "seronegative" for both autoantibodies (RF and ACPA), while 405 had at least 1 positive test from RF and CCP tests. Twentyone patients could not be classified according to ACR EULAR 2010 criteria due to missing data (i.e., 3 had sufficient criteria even without information regarding serologic status to be classified having RA). From these 792 patients, 645 (79.3\%) were classified RA, 399 (61.9\%) of them being seropositive, and 246 (38.1\%) seronegative. From the 147 not being classified RA, 21 (14.3\%) were seropositive and 126 (85.7\%) seronegative (Fig. 1). We included 172 patients in the current study $(n=58$ had not received a csDMARD during the first year, $n=19$ were lost to follow-up, $n=29$ had missing data). Most characteristics were similar between excluded and included patients, except for baseline HAQ-DI, which was slightly lower for excluded than included patients (mean \pm SD $0.89 \pm 0.67$ vs $1.1 \pm 0.68$ ). Baseline characteristics are shown in Table 1 . The mean $\pm \mathrm{SD}$ age was $49.5 \pm 12.8$ years, and $80.8 \%$ of patients were females. At inclusion, the mean \pm SD number of swollen joints was $9.0 \pm 5.5$ and number of tender joints $11.7 \pm 7.1$. Mean DAS28 score at baseline was $5.5 \pm 1.1$. MTX was the most commonly prescribed first DMARD (98/172 [57\%] patients).

\section{Predictors of good or moderate EULAR response at 12 months}

At 1 year, 114/172 (66\%) patients showed a good or moderate EULAR response. On univariate analysis, a good or moderate EULAR response was significantly associated with swollen joint count $(\geq 7)$, early treatment (started within 3 months after the date of first reported 


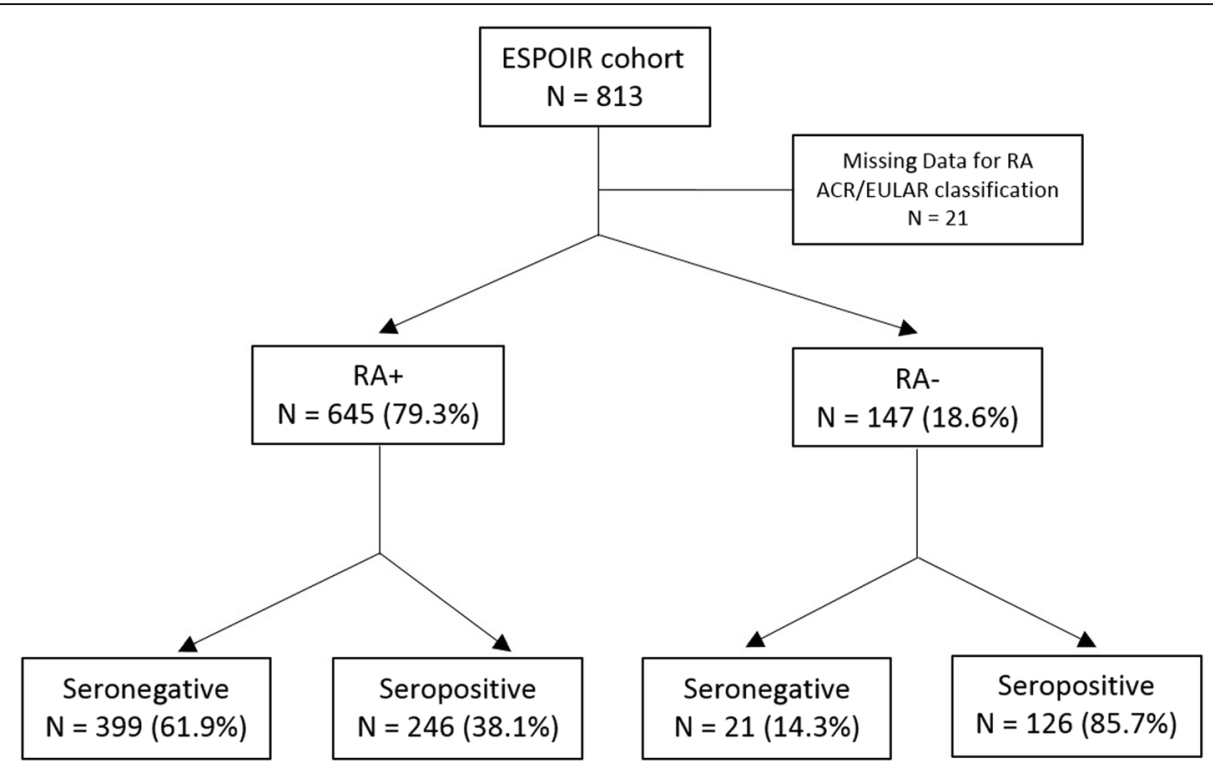

Fig. 1 Flowchart of ESPOIR cohort's patients. RA, rheumatoid arthritis; RA+, ACR/EULAR classification for RA fulfilled; RA-, ACR/EULAR classification for RA not fulfilled; seronegative, both rheumatoid factor- and CCP-tests negative; seropositive, at least 1 positive test from rheumatoid factorand CCP-tests

synovitis), ESR, CRP level, and HAQ-DI $\geq 1$ ( $p<0.05$ for all comparisons).

On multivariable analysis (Table 2), a good or moderate EULAR response was associated with only early use (within 3 months) of csDMARDs (OR $2.41[95 \% \mathrm{CI}$ $1.07-5.42], p=0.03)$.

\section{Association between early therapeutic response to MTX and LEF versus other csDMARDs (hydroxychloroquine, sulfasalazine)}

After adjustment for propensity of patients to receive MTX, LEF, or the 2 drugs combined versus other csDMARDs, we found no significant difference in response rates at 1-year follow-up visit (Additional file 1: Table S1).

\section{Predictors of radiographic outcome at $\mathbf{1 2}$ months}

Radiographic data were available for 149 patients at 1 year. In total, $15(10 \%)$ and $9(6 \%)$ showed a progression of at least 1 point and 5 points, respectively, in mTSS at 1 year. On multivariable analysis, the probability of radiographic progression by a least 1 mTSS point at 1 year was significantly increased in patients with erosions on baseline radiographic assessment and was decreased in those with $>10$ tender joints. The only characteristic associated with a radiographic progression of at least 5 mTSS points at 1 year was the presence of erosions at baseline ( $\mathrm{OR}=5.42[95 \%$ CI 1.14-25.7], $p=0.03$ ).

\section{Factors associated with functional disability}

HAQ-DI data were available for 150 patients at 1 year. HAQ-DI was $>0.5$ for $72(48 \%)$ patients at 1 year. On multivariable analysis, functional disability (defined by a HAQ-DI $>0.5$ ) at 1 year was significantly associated with increased baseline functional disability defined as HAQ-DI $>1$ (OR $=6.59$ [95\% CI 3.29-13.2], $p<0.001)$, female sex $(0.28[0.10-0.79], p=0.02)$, ESR $>15(0.45$ [0.20-0.98], $p=0.05)$, and active smoking status (2.59 [1.00-6.69], $p=0.05)$ [20].

\section{Results in entire ESPOIR cohort and alternative outcome measures}

The sensitivity analysis in the entire ESPOIR cohort was applied to the 522 patients fulfilling the aforementioned selection criteria, with 69 having missing data for at least 1 critical variable in our analysis plan and 453 thus finally included. The EULAR good/moderate response was obtained by $369(81.5 \%)$ of these included patients. The main result was confirmed in this larger sample of patients, with a significant association between the early DMARD start and a favorable therapeutic response: OR 2.29 [1.27-4.12], $p=0.006$. The larger sample size revealed another significantly associated variable, with patients having a higher CRP $(\geq 7 \mathrm{mg} / \mathrm{L})$ also showing a higher likelihood of favorable outcome: OR 1.83 [1.132.97], $p=0.015$. Interestingly, the addition of the variable "seronegative yes/no" in the list of candidate variables when building the multivariate logistic model showed no relevant association with the therapeutic outcome (variable not retained by the stepwise model). When other 
Table 1 Baseline characteristics of the study population $(n=$ 172)

\begin{tabular}{|c|c|}
\hline Characteristic & \\
\hline Women, no./total (\%) & 139/172 (80.8) \\
\hline Age, mean $\pm S D$, years & $49.5 \pm 12.8$ \\
\hline \multicolumn{2}{|l|}{ Smoking status, no. (\%) } \\
\hline Current smoking & $46(26.7)$ \\
\hline Past smoking & $42(24.4)$ \\
\hline Never smoked & $93(54.1)$ \\
\hline \multicolumn{2}{|l|}{ DAS28, no. (\%) } \\
\hline Low disease activity & $1(0.6)$ \\
\hline Moderate disease activity & $70(40.7)$ \\
\hline High disease activity & $101(58.7)$ \\
\hline Tender joint count, mean \pm SD & $9 \pm 5.5$ \\
\hline Swollen joint count, mean $\pm S D$ & $11.7 \pm 7.1$ \\
\hline HAQ-DI, mean \pm SD & $1.1 \pm 0.7$ \\
\hline $\mathrm{ESR}(\mathrm{mm} / \mathrm{h})$, mean $\pm \mathrm{SD}$ & $25.2 \pm 23.6$ \\
\hline $\mathrm{CRP}(\mathrm{mg} / \mathrm{dl})$, mean $\pm \mathrm{SD}$ & $23.2 \pm 42.8$ \\
\hline mTSS, mean $\pm S D$ & $5.5 \pm 7.0$ \\
\hline \multicolumn{2}{|l|}{ Treatment, no. (\%) } \\
\hline Methotrexate & $98(57)$ \\
\hline Leflunomide & $12(7)$ \\
\hline Sulfasalazine & $22(12.8)$ \\
\hline Hydroxychloroquine & $37(21.5)$ \\
\hline Cyclophosphamide & $1(0.6)$ \\
\hline Biologic DMARD & $2(1.2)$ \\
\hline
\end{tabular}

CRP C-reactive protein, DAS28 Disease Activity Score in 28 joints, DMARD disease-modifying antirheumatic drug, ESR erythrocyte sedimentation rate, HAQ Health Assessment Questionnaire-Disability Index, mTSS van der Heijdemodified total Sharp score

outcome measures of the therapeutic response were applied to the seronegative patients (DAS28 remission or DAS28 remission/low disease activity), the association with the early DMARD start remained in the final multivariate model, although at a statistically non-significant level (data not shown).

\section{Discussion}

With an inception cohort of patients with early inflammatory arthritis (the ESPOIR cohort), we described the characteristics and evolution over the first year of follow-up of those fulfilling the ACR/EULAR criteria for RA but with no detectable RF or anti-CCP antibodies ("seronegative RA"). Overall, 246 of the 803 included patients fulfilled criteria for RA despite a seronegative status. The most important predictor of good therapeutic response in these patients was the early introduction (within 3 months after symptom onset) of a csDMARD (MTX being the most commonly used). Response rates did not differ across available csDMARDs after
Table 2 Multivariable analysis of factors associated with good or moderate EULAR response at 1 year $(n=172)$

\begin{tabular}{llll}
\hline & OR & $95 \% \mathrm{Cl}$ & $p$ \\
\hline Age $>$ 49 years & 0.82 & $0.35-1.89$ & 0.64 \\
Sex (female) & 1.52 & $0.51-4.53$ & 0.45 \\
Education level & & & \\
$\quad$ Primary school & 0.29 & $0.08-1.03$ & 0.06 \\
$\quad$ Middle school & 0.53 & $0.19-1.46$ & 0.22 \\
$\quad$ High school & ref & - & 0.23 \\
$\quad$ University & 0.73 & $0.25-2.15$ & 0.56 \\
Smoking status & & & \\
$\quad$ Current smoking & ref & - & 0.70 \\
$\quad$ Past smoking & 0.82 & $0.24-2.77$ & 0.75 \\
$\quad$ Never smoked & 1.23 & $0.42-3.65$ & 0.71 \\
DAS28 level & & & 0.71 \\
Tender joint count $\geq 10$ & 1.21 & $0.47-3.14$ & 0.68 \\
Swollen joint count $\geq 7$ & 0.94 & $0.33-2.69$ & 0.91 \\
Rheumatologist global assessment $\geq 66 / 100$ (VAS) & 1.15 & $0.51-2.58$ & 0.74 \\
Early DMARD treatment ${ }^{1}$ & 2.41 & $1.07-5.42$ & 0.03 \\
HAQ-DI $\geq 1$ & 1.41 & $0.67-2.98$ & 0.37 \\
ESR $\geq 15$ mm/h & 1.42 & $0.66-3.03$ & 0.37 \\
CRP level $\geq 7$ mg/l & 0.71 & $0.32-1.61$ & 0.42 \\
mTSS (per unit) & 1.00 & $0.94-1.07$ & 0.96 \\
mTSS & 0.87 & $0.30-2.52$ & 0.79 \\
\hline CRPC-reative & & & \\
& & &
\end{tabular}

CRP C-reactive protein, DAS28 Disease Activity Score in 28 joints, ESR erythrocyte sedimentation rate, HAQ-DI Health Assessment QuestionnaireDisability Index, mTSS van der Heijde-modified total Sharp score, VAS visual analog scale, OR odds ratio, 95\% Cl 95\% confidence interval

${ }^{1}$ Within 3 months after the first joint swelling

adjustment for potential baseline prognostic factors and thus expected confounding by indication bias.

According to common therapeutic recommendations [21], MTX was the first-line therapy for most patients in our cohort (57\%), despite being negative for serologic factors of RA, which are considered relevant elements for diagnostic certainty and potentially severe prognosis. With regard to other similar studies, MTX was often used as first-line treatment [22, 23], which is probably due to the time frames, the patients in our study cohort having been enrolled more recently (years 2002-2005).

Overall, $66 \%$ of our patients had a good or moderate EULAR response to the first DMARD used at 1-year follow-up. Although we cannot directly compare to previous studies because of different outcomes used and potentially different inclusion criteria or recruitment methods, previous studies also showed a favorable therapeutic response to traditionally used medications in seronegative RA patients [22, 24, 25].

With regard to factors associated with a more favorable outcome and independent of other measurable and collectable characteristics, the only relevant prognostic 
marker of a good therapeutic response was the early introduction (within 3 months after symptom onset) of the DMARD. This finding was already reported for the entire ESPOIR cohort [19], which confirms that the serologic status of a patient should not imply a major change in therapeutic management, provided the diagnostic approach has been appropriately conducted. Other studies have shown similar results and confirmed the benefit of early treatment start, but our study is the first time that this finding was replicated in seronegative patients [26-29].

Because seronegativity is supposed to be associated with better overall prognosis of RA, we expected that these patients would receive less intensive treatment than patients with detectable levels of RF and/or anti-CCP antibodies [30-32]. However, a similar proportion of patients had received MTX or LEF as first-line therapy in the entire ESPOIR cohort as in this sub-population with no detectable serological biomarker of the disease [19]. Other csDMARDs were also alternatively prescribed in these conditions, and we investigated whether this prescription might have measurable consequences in short-term prognosis (first-year therapeutic response in particular). After adjustment for known and collected factors associated with potentially worse prognosis and thus a higher propensity for the rheumatologist to prescribe more intensive treatments, the response rates did not differ in our patients with seronegative RA who received anchor drugs or "second-choice drugs" such as sulfasalazine or hydroxychloroquine. However, the method applied (propensityscore adjustment) as well as the limited number of patients in the second group requires cautious interpretation, and we report our observations without drawing any definite conclusions regarding the optimal choice of the first csDMARD in this context. In another study, a similar response rate was also reported in anti-CCP-negative RA patients receiving MTX or a combination of csDMARDs [7].

\section{Conclusions}

In terms of prognostic factors associated with RA structural progression, we confirmed that the strongest predictor of further joint degradation remains the presence of early erosions seen on hands and feet radiographs [33]. These results confirm that despite the overall better expected prognosis with seronegative versus seropositive RA, in particular anti-CCP positivity, careful and complete examination of every patient is crucial, before the appropriate and optimal therapeutic management can be determined.

Several limitations of our study must be acknowledged. First, because our main objective was to determine factors associated with a favorable therapeutic response in seronegative RA, we restricted our analyses to patients who received a csDMARD over the follow-up period. In the ESPOIR cohort, 246 patients had seronegative RA according to ACR/EULAR criteria, but 58 did not receive any DMARD during the first year of follow-up and were thus not included. Presumably for these patients, their rheumatologist considered that they had sufficiently mild disease or potentially self-remitting disease to avoid a specific drug prescription on the basis of clinical, biological, or radiological data and short-term disease course. Therefore, our results should be applied to patients fulfilling RA criteria and justifying DMARD initiation based on the rheumatologist's opinion. Indeed, the observed disease activity was higher on average in our population than in other reported cohorts of seronegative RA patients $[7,22,24,25]$. It is important to note that for fulfilling ACR/EULAR 2010 RA criteria, patients with seronegative RA should have a higher number of joints involved than seropositive patients, though high disease activity is an important prognostic factor in early arthritis, and thus a potential confounding factor in our work.

In conclusion, we confirmed that seronegative RA does not greatly differ from overall RA in terms of both therapeutic response and structural and functional prognosis and, most importantly, that the usual and consensually recommended concepts of therapeutic management should not be applied differently in these patients. Indeed, despite a generally more favorable expected prognosis with seronegative RA, the positive impact of an appropriate initial management remains measurable, with the concept of the "window of opportunity" being confirmed in this sub-population, pleading for an early start of a DMARD, ideally within 3 months after symptom onset.

\section{Supplementary information}

Supplementary information accompanies this paper at https://doi.org/10. 1186/s13075-019-2020-x.

Additional file 1: Table S1. Impact of the type of prescribed diseasemodifying anti-rheumatic drug (DMARD) on 1-year EULAR response rate: multivariable analyses adjusted for the propensity of receiving methotrexate or leflunomide as first-line therapy.

\section{Abbreviations}

ACPA: Anti-citrullinated peptide antibodies; ACR/EULAR: American College of Rheumatology/European League Against Rheumatism; CRP: C-reactive protein; DAS28: Disease Activity Score in 28 joints; csDMARDs: Conventional synthetic disease-modifying antirheumatic drugs; ESR: Erythrocyte sedimentation rate; HAQ-DI: Health Assessment Questionnaire-Disability Index; LEF: Leflunomide; mTSS: Van der Heijde-modified total Sharp score; MTX: Methotrexate; NSJ: Number of swollen joints; NTJ: Number of tender joints; RA: Rheumatoid arthritis; RF: Rheumatoid factor; VAS: Visual analog scale

\section{Acknowledgements}

We thank the French rheumatologists who referred their patients to the ESPOIR cohort in the following rheumatology departments: Amiens (P. 
Fardellone, P. Boumier), Bordeaux (T. Schaeverbeke), Brest (A. Saraux), Lille (R.M. Flipo), Montpellier (B. Combe), Paris-Bicêtre (X. Mariette), Paris-Bichat (O. Meyer), Paris-Cochin (M. Dougados), Paris-La Pitié (B. Fautrel), Paris-St Antoine (F. Berenbaum), Rouen (X. Le Loët, O. Vittecoq), Strasbourg (J. Sibilia), Toulouse (A. Cantagrel), and Tours (P. Goupille).

We are grateful to N. Rincheval for data management and expert monitoring;

\section{Authors' contributions}

$\mathrm{CL}$ analyzed and interpreted the data and reviewed the manuscript critically. $J M a$ interpreted the data and wrote the manuscript. MD, CD, JMo, AC, BC and BF critically reviewed the protocol of the study. All authors read and approved the final manuscript.

\section{Funding}

An unconditional grant from Merck (MSD) is allocated for the first 3 years for the constitution of the cohort. No specific funding for the current analysis.

\section{Availability of data and materials}

The data that support the findings of this study are available from the ESPOIR scientific committee but restrictions apply to the availability of these data, which were used under license for the current study, and so are not publicly available. Data are however available from the authors upon reasonable request and with permission of the ESPOIR scientific committee.

\section{Ethics approval and consent to participate}

The protocol of the study was approved in July 2002 by the Ethics Committee of Montpellier University (no. 020307). All patients gave their signed informed consent to be in the study.

\section{Consent for publication}

Not applicable.

\section{Competing interests}

The authors declare that they have no competing interests.

\section{Author details}

${ }^{1}$ Rheumatology Department, CHU Montpellier, Montpellier University, Montpellier, France. ${ }^{2}$ Rheumatology Department, CHU Martinique, Pierre Zobda-Quitman Hospital, 97200 Fort-de-France, Martinique, French West Indies, France. ${ }^{3}$ Rheumatology Department, University Paul Sabatier Toulouse III, Purpan Hospital, 31059 Toulouse, France. ${ }^{4}$ Sorbonne Université, Institut Pierre Louis d'Epidémiologie et Santé Publique, INSERM UMR S 1136, AP-HP, Groupe hospitalier Pitié Salpêtrière, Service de Rhumatologie, Paris, France.

\section{Received: 25 June 2019 Accepted: 1 October 2019}

\section{Published online: 15 November 2019}

\section{References}

1. Guillemin F, Saraux A, Guggenbuhl P, Roux CH, Fardellone P, Le Bihan E, et al. Prevalence of rheumatoid arthritis in France: 2001. Ann Rheum Dis, 2005;64(10):1427-30

2. Mouterde G, Lukas C, Goupille P, et al. Association of anticyclic citrullinated peptide antibodies and/or rheumatoid factor status and clinical presentation in early arthritis: results from the ESPOIR Cohort. J Rheumatol. 2014;41:1614-22.

3. Vallbracht I, Rieber J, Oppermann M, Forger F, Siebert U, Helmke K, et al. Diagnostic and clinical value of anti-cyclic citrullinated peptide antibodies compared with rheumatoid factor isotypes in rheumatoid arthritis. Ann Rheum Dis. 2004;63:1079-84.

4. Mewar D, Coote A, Moore DJ, Marinou I, Keyworth J, Dickson MC, et al. Independent associations of anti-cyclic citrullinated peptide antibodies and rheumatoid factor with radiographic severity of rheumatoid arthritis. Arthritis Res Ther. 2006;8:R128.

5. Kastbom A, Strandberg G, Lindroos A, et al. Anti-CCP antibody test predicts the disease course during 3 years in early rheumatoid arthritis (the Swedish TIRA project). Ann Rheum Dis. 2004;63:1085-9.

6. Nell VP, Machold KP, Stamm TA, et al. Autoantibody profiling as early diagnostic and prognostic tool for rheumatoid arthritis. Ann Rheum Dis. 2005;64:1731-6.
7. van den Broek $M$, Dirven $L$, Klarenbeek NB, et al. The association of treatment response and joint damage with ACPA-status in recent-onset RA: a subanalysis of the 8-year follow-up of the BeSt study. Ann Rheum Dis. 2012;71:245-8.

8. Combe B, Landewe R, Lukas C, et al. EULAR recommendations for the management of early arthritis: report of a task force of the European Standing Committee for International Clinical Studies Including Therapeutics (ESCISIT). Ann Rheum Dis. 2007;66:34-45.

9. Smolen JS, Landewe R, Breedveld FC, et al. EULAR recommendations for the management of rheumatoid arthritis with synthetic and biologic diseasemodifying antirheumatic drugs. Ann Rheum Dis. 2010;69:964-75.

10. Zink A, Listing J, Nieworth M, et al. The national database of the German collaborative arthritis centres: II-treatment of patients with rheumatoid arthritis. Ann Rheum Dis. 2001;60:207-13.

11. Benefit of early treatment in inflammatory polyarthritis patients with anticyclic citrullinated peptide antibodies versus those without antibodies. Arthritis Care Res. Arthritis Care Res. 2010;62:664-75.

12. Barra L, Pope JE, Orav JE, et al. Prognosis of seronegative patients in a large prospective cohort of patients with early inflammatory arthritis. J Rheumatol. 2014:2361-9.

13. da Mota LM, Dos Santos Neto LL, de Carvalho JF, Pereira IA, Burlingame $R$ Ménard HA, et al. The presence of anti-citrullinated protein antibodies (ACPA) and rheumatoid factor on patients with rheumatoid arthritis (RA) does not interfere with the chance of clinical remission in a follow-up of 3 years. Rheumatol Int. 2012;12:3807-12.

14. Combe B, Benessiano J, Berenbaum F, et al. The ESPOIR cohort: a ten-year follow-up of early arthritis in France: methodology and baseline characteristics of the 813 included patients. Joint Bone Spine. 2007;5:440-5.

15. Aletaha D, Neogi T, Silman A, et al. Rheumatoid arthritis classification criteria: an America College of Rheumatology/European League Against Rheumatism collaborative iniiative. Ann Rheum Dis. 2010;69:1580-8.

16. Prevoo ML, van 't Hof MA, Kuper HH, van Leeuwen MA, van de Putte LB, van Riel PL. Modified disease activity scores that include twenty-eightjoint counts. Development and validation in a prospective longitudinal study of patients with rheumatoid arthritis. Arthritis Rheum. 1995;38:44-8.

17. Guillemin F, Braincon S, Pourel J. [Measurement of the functional capacity in rheumatoid polyarthritis: a French adaptation of the Health Assessment Questionnaire (HAQ)]. [Article in French]. Rev Rhum Mal Osteoartic. 1991;58: 459-65.

18. van der Heijde DM, van Riel PL, Nuver-Zwart IH, Gribnau FW, vad de Putte LB. Effects of hydroxychloroquine and sulphasalazine on progression of joint damage in rheumatoid arthritis. Lancet. 1989;333:1036-1038.

19. Lukas C, Combe B, Ravaud P, Sibilia J, Landew R, van der Heijde D. Favorable effect of very early disease-modifying antirheumatic drug treatment on radiographic progression in early inflammatory arthritis: data from the Étude et Suivi des polyarthrites indifférenciées récentes (study and followup of early undifferentiated polyarthritis). Arthritis Rheum. 2011;63(7):1804-11.

20. Maska L, Anderson J, Michaud K. Measures of functional status and quality of life in rheumatoid arthritis: Health Assessment Questionnaire Disability Index (HAQ), Modified Health Assessment Questionnaire (MHAQ), Multidimensional Health Assessment Questionnaire (MDHAQ), Health Assessment Questionnaire II (HAQ-II), Improved Health Assessment Questionnaire (Improved HAQ), and Rheumatoid Arthritis Quality of Life (RAQoL). Arthritis Care Res. 2011;63(Suppl 11):S4-13.

21. Smolen JS, Landewé R, Bijlsma J, Burmester G, Chatzidionysiou K, Dougados $M$, et al. EULAR recommendations for the management of rheumatoid arthritis with synthetic and biological disease-modifying antirheumatic drugs: 2016 update. Ann Rheum Dis. 2017;76(6):960-77.

22. Farragher TM, Lunt M, Plant D, Bunn DK, Barton A, Symmons DPM. Benefit of early treatment in inflammatory polyarthritis patients with anti-cyclic citrullinated peptide antibodies versus those without antibodies. Arthritis Care Res. 2010;62(5):664-75.

23. Kastbom A, Strandberg G, Lindroos A, Skogh T. Anti-CCP antibody test predicts the disease course during 3 years in early rheumatoid arthritis (the Swedish TIRA project). Ann Rheum Dis. 2004;63(9):1085-9.

24. Barra L, Pope JE, Orav JE, Boire G, Haraoui B, Hitchon C, et al. Prognosis of seronegative patients in a large prospective cohort of patients with early inflammatory arthritis. J Rheumatol. 2014;41(12):2361-9.

25. Ursum J, Bos WH, van Dillen N, Dijkmans BA, van Schaardenburg D. Levels of anti-citrullinated protein antibodies and IgM rheumatoid factor are not 
associated with outcome in early arthritis patients: a cohort study. Arthritis Res Ther. 2010;12(1):R8.

26. Boers M. Rheumatoid arthritis. Treatment of early disease. Rheum Dis Clin North Am. 2001;27(2):405-14 x.

27. Möttönen T, Hannonen P, Korpela M, Nissilä M, Kautiainen H, llonen J, et al. Delay to institution of therapy and induction of remission using single-drug or combination-disease-modifying antirheumatic drug therapy in early rheumatoid arthritis. Arthritis Rheum. 2002;46(4):894-8.

28. Verstappen SMM, Jacobs JWG, Bijlsma JWJ, Heurkens AHM, van BoomaFrankfort C, Borg EJT, et al. Five-year followup of rheumatoid arthritis patients after early treatment with disease-modifying antirheumatic drugs versus treatment according to the pyramid approach in the first year. Arthritis Rheum. 2003;48(7):1797-807.

29. Emery P. Evidence supporting the benefit of early intervention in rheumatoid arthritis. J Rheumatol Suppl. 2002:66:3-8.

30. Katchamart W, Koolvisoot A, Aromdee E, Chiowchanwesawakit P, Muengchan C. Associations of rheumatoid factor and anti-citrullinated peptide antibody with disease progression and treatment outcomes in patients with rheumatoid arthritis. Rheumatol Int. 2015;35(10):1693-9.

31. Truchetet M-E, Dublanc S, Barnetche T, Vittecoq O, Mariette X, Richez C, et al. Association of the presence of anti-carbamylated protein antibodies in early arthritis with a poorer clinical and radiologic outcome: data from the French ESPOIR Cohort. Arthritis Rheumatol Hoboken NJ. 2017:69(12):2292-302.

32. Santos-Moreno P, Sánchez G, Castro C. Rheumatoid factor as predictor of response to treatment with anti-TNF alpha drugs in patients with rheumatoid arthritis: Results of a cohort study. Medicine (Baltimore). 2019; 98(5):e14181.

33. Dixey J, Solymossy C, Young A, Early RA. Study. Is it possible to predict radiological damage in early rheumatoid arthritis (RA)? A report on the occurrence, progression, and prognostic factors of radiological erosions over the first 3 years in 866 patients from the Early RA Study (ERAS). J Rheumatol Suppl. 2004:69:48-54.

\section{Publisher's Note}

Springer Nature remains neutral with regard to jurisdictional claims in published maps and institutional affiliations.

Ready to submit your research? Choose BMC and benefit from:

- fast, convenient online submission

- thorough peer review by experienced researchers in your field

- rapid publication on acceptance

- support for research data, including large and complex data types

- gold Open Access which fosters wider collaboration and increased citations

- maximum visibility for your research: over $100 \mathrm{M}$ website views per year

At $\mathrm{BMC}$, research is always in progress.

Learn more biomedcentral.com/submissions 